\title{
Simulation-based Machine Learning for Optoelectronic Device Design: Perspectives, Problems, and Prospects
}

Joachim Piprek ( $\square$ piprek@nusod.org )

NUSOD Institute LLC https://orcid.org/0000-0002-4002-1629

\section{Research Article}

Keywords: machine learning, deep learning, neural networks, artificial intelligence, numerical simulation, design optimization, light-emitting diode

Posted Date: March 5th, 2021

DOI: https://doi.org/10.21203/rs.3.rs-267857/v1

License: (9) This work is licensed under a Creative Commons Attribution 4.0 International License. Read Full License

Version of Record: A version of this preprint was published on March 24th, 2021. See the published version at https://doi.org/10.1007/s11082-021-02837-8. 


\section{Abstract}

Numerical simulation and machine learning represent opposite approaches to computational analysis of the real world, deductive vs. inductive. However, both methods suffer from various uncertainties and even their combination often fails to link theory and reality. Focusing on GaN-based light-emitting diode (LED) design optimization, this paper evaluates examples of simulation-based machine learning from a physics point of view. Strategies are suggested for achieving more realistic predictions.

\section{Main Text}

Computer simulations embed theoretical models into a practical environment (Piprek 2017). This enables a realistic test of such models by comparing calculated results to measurements. Simulations can thereby help explain experimental results that would otherwise be hard to understand. Simulations also allow for performance predictions for novel devices. However, it is well known that initial simulation results hardly ever agree with measurements. In other words, computer simulations often fail to represent the real world and create a virtual reality instead in which artificial effects may happen (Fig. 1). This is not surprising as mathematical models always simplify reality. There are different levels of simplification, from short analytical formulas to complex systems of equations, which are all based on specific assumptions about relevant physical processes. Certain assumptions may be inappropriate in a practical situation. Contradicting assumptions may even deliver almost identical results (Piprek 2015). Another problem is the employment of unrealistic material parameters. Published values of such parameters often vary substantially (Müller et al. 2014, Piprek et al. 2015). Careful adjustments of computer simulations are required to find agreement between theory and reality (Piprek et al. 2002, Wasmer et al. 2017).

Machine learning, on the other hand, usually collects data in the real world and performs statistical analyses (Fig. 1). This is especially valuable when the amount of data is very large and hard to digest. Deep learning is currently the most popular machine learning method (LeCunn et al. 2015) and it is based on multi-layered artificial neural networks (ANNs). Many data sets are needed to train an ANN. Due to scarcity and scatter of real-world data, experimental data collection is often replaced by computer simulations based on established theories. Such physics-based machine learning methods are increasingly utilized in materials science (Schmidt et al. 2019). Various semiconductor material systems are explored and optimized for applications in optoelectronics (Lu et al. 2019, Liu et al. 2020, Luo et al. 2020). Simulation-based machine learning is also popular with photonic devices (Molesky et al. 2018, Ma et al. 2020, Genty et al. 2020) utilizing solutions to Maxwell's equations that involve only few material parameters. Compared to photonics, optoelectronic devices are much more complex as they combine optical, electronic, and thermal processes. As indicated above, computer simulations of such devices not only involve various modeling approximations in need of validation, but also many uncertain material parameters. Simulation-based machine learning has been applied to various optoelectronic devices (Cicic \& Tomic 2019, Hakimian et al. 2020, Ma \& Li 2020, Wagner-Mohnsen \& Altermatt 2020, Razzaq et al. 2020, Majeed et al. 2020). Light-emitting diodes (LEDs) in particular have been a target of machne 
learning (Zhu et al. 2012, Kim et al. 2015, Rouet-Leduc et al. 2016, Janai et al. 2018, Fan et al. 2020, Ibrahim et al. 2020).

In the following, we evaluate a few of these machine-learning applications that are focused on GaNbased LEDs. These blue light emitters have been receiving great attention in recent years due to their widespread utilization in lighting, displays and other fields (Wasisto et al. 2019). Countless and partially contradicting GaN-LED simulation studies have been published, with the LED efficiency being in the center of interest in most cases (Piprek 2010, Verzellesi et al. 2013, Usman et al. 2020). Not surprisingly, one of the first machine learning applications to GaN-LEDs is focused on efficiency improvements (Zhu et al. 2012). The authors employ a genetic algorithm to select combinations of basic semiconductor layer structures with random material composition, thickness, and doping. Utilizing a Matlab-based LED model, the design is successively improved to maximize an efficiency-related fitness function. After about 500 generations with a typical population size of 1000 , an optimum active region doping scheme is identified that enhances the efficiency by limiting the electron leakage from the InGaN quantum wells (QWs). A further leakage reduction is discovered for a novel design of the electron blocking layer (EBL). The EBL is split up into 10 individual 3nm-thick layers with different composition. However, this optimization strategy ignores the strong influence of band-offset and EBL doping reported earlier (Piprek \& Li 2010).

EBL doping optimization is included in a follow-up paper which now splits the EBL into $1 \mathrm{~nm}$-thin AlGalnN sub-layers of different compositions and doping densities (Kim et al. 2015). The genetic algorithm identifies an optimum EBL design with uniform Mg doping density of $10^{19} \mathrm{~cm}^{-3}$, which is the maximum of the doping range considered, in agreement with earlier findings (Piprek \& Li 2010). An internal quantum efficiency (IQE) of 0.59 is predicted for the optimized LED design at $200 \mathrm{~A} / \mathrm{cm}^{2}$ current density, which is a significant improvement compared to IQE $=0.43$ with the reference LED design. IQE represents the ratio between the number of photons generated inside the QWs to the number of electrons injected into the device. It thereby accounts for carrier losses caused by non-radiative recombination or leakage (Piprek 2010).

For evaluation and validation, we simulate the same LED structures utilizing APSYS by Crosslight Software. This widely used code self-consistently computes carrier transport, the wurtzite electron band structure of strained QWs, and photon emission. Schrödinger and Poisson equations are solved iteratively in order to account for the QW deformation with changing device bias (quantum-confined Stark effect). The transport model includes drift and diffusion of electrons and holes, Fermi statistics, built-in polarization, and thermionic emission at hetero-interfaces, as well as all relevant radiative and nonradiative recombination mechanisms. In agreement with the reference papers, self-heating is excluded from our study. A more detailed discussion of LED device models is published elsewhere (Piprek \& Li 2005, Piprek 2020). The layer structure of the reference LED is given in Table 1 (Kim et al. 2015). The vertical band gap profiles are plotted in Fig. 2 for the reference LED (red line) and for two optimized designs.

Table. 1: Layer structure of the reference LED (EBL - electron blocking layer). 


\begin{tabular}{|llll|}
\hline Layer & Composition & Doping & Thickness \\
\hline p-cladding & GaN:Mg & $1^{\prime} 10^{19} \mathrm{~cm}^{-3}$ & $200 \mathrm{~nm}$ \\
\hline p-EBL & $\mathrm{Al}_{0.19} \mathrm{Ga}_{0.81} \mathrm{~N}: \mathrm{Mg}$ & $1^{\prime} 10^{19} \mathrm{~cm}^{-3}$ & $20 \mathrm{~nm}$ \\
\hline p-injector & GaN:Mg & $1^{\prime} 10^{19} \mathrm{~cm}^{-3}$ & $4 \mathrm{~nm}$ \\
\hline spacer & $\mathrm{GaN}$ & & $6 \mathrm{~nm}$ \\
\hline quantum well & $\mathrm{In}_{0.15} \mathrm{Ga}_{0.85} \mathrm{~N}$ & & $3 \mathrm{~nm}$ \\
\hline n-barrier & $\mathrm{GaN}: \mathrm{Si}$ & $5^{\prime} 10^{18} \mathrm{~cm}^{-3}$ & $10 \mathrm{~nm}$ \\
\hline quantum well & $\mathrm{In}_{0.15} \mathrm{Ga}_{0.85} \mathrm{~N}$ & & $3 \mathrm{~nm}$ \\
\hline n-barrier & $\mathrm{GaN}: \mathrm{Si}$ & $5^{\prime} 10^{18} \mathrm{~cm}^{-3}$ & $10 \mathrm{~nm}$ \\
\hline quantum well & $\mathrm{In}_{0.15} \mathrm{Ga}_{0.85} \mathrm{~N}$ & & $3 \mathrm{~nm}$ \\
\hline n-barrier & $\mathrm{GaN}_{\mathrm{Si}}$ & $5^{\prime} 10^{18} \mathrm{~cm}^{-3}$ & $10 \mathrm{~nm}$ \\
\hline quantum well & $\mathrm{In}_{0.15} \mathrm{Ga}_{0.85} \mathrm{~N}$ & & $3 \mathrm{~nm}$ \\
\hline n-barrier & $\mathrm{GaN}_{\mathrm{Si}}$ & $5^{\prime} 10^{18} \mathrm{~cm}^{-3}$ & $10 \mathrm{~nm}$ \\
\hline quantum well & $\mathrm{In}_{0.15} \mathrm{Ga}_{0.85} \mathrm{~N}$ & & $3 \mathrm{~nm}$ \\
\hline n-cladding & $\mathrm{GaN}_{\mathrm{Si}}$ & $7^{\prime} 10^{18} \mathrm{~cm}^{-3}$ & $200 \mathrm{~nm}$ \\
\hline
\end{tabular}

The calculated LED efficiency is known to strongly depend on material parameters such as Auger recombination coefficient (Piprek et al. 2015), built-in polarization (Piprek \& Li 2013), band offset at hetero-junctions (Piprek \& Li 2013), and hole mobility, none of which is specified by Kim et al. (2015). In order to reproduce the reference IQE characteristic, we here assume an Auger coefficient of $C=10^{-30} \mathrm{~cm}^{6} / \mathrm{s}$ as well as a conduction band offset ratio of 0.6 and a polarization screening factor of 0.5 , all within the range of published values. However, the hole mobility is seldom mentioned. Lower hole mobility leads to enhanced electron leakage into the p-doped layers, thereby reducing the efficiency. The solid lines in Fig. 3 demonstrate the influence of the hole mobility on the simulated IQE characteristic. A typical hole mobility of $1 \mathrm{~cm}^{2} /$ Vs produces about $1 \%$ electron leakage at $200 \mathrm{~A} / \mathrm{cm}^{2}$ current density (blue line in Fig. 3). A 10times lower hole mobility gives $25 \%$ leakage and it moves our results close to the IQE reported by Kim et al. (2015) (red line in Fig. 3). The optimized LED structure promises improved electron blocking, as suggested by the blue bandgap profile in Fig. 2. Indeed, this LED design produces a somewhat higher efficiency by reducing the electron leakage to $19 \%$ (red dashed line in Fig. 3). But we are unable to confirm the complete leakage elimination or the strong efficiency improvement predicted in the reference paper, even after testing alternative parameter combinations. Likely reasons are differences between other material parameters and/or between the underlying models. As illustrated in Fig. 3, modeling 
uncertainties produce a substantial error range in the simulation, which is larger than the efficiency improvement achieved by the design optimization at $200 \mathrm{~A} / \mathrm{cm}^{2}$. Simulation models and parameters should be first calibrated by comparison to relevant measurements of the reference device (Piprek et al. 2002). This is often a time-consuming process but it is a prerequisite for reliable performance predictions.

However, such continuum models ignore the atomistic structure of very thin layers. The optimized LED structure of Kim et al. (2015) features a $1 \mathrm{~nm}$ thick AlGalnN layer with only $5 \%$ indium content inside the EBL (blue dashed line in Fig. 2), for which the average lateral distance of indium atoms is substantially larger than the layer thickness. In other words, the assumption of an uniform energy barrier is incorrect. What is more, such thin barrier enables carrier tunneling which is not considered in these simulations. Thus, the machine learning results clearly overstep the validity limits of the underlying LED simulations. Design space boundaries should be carefully evaluated and strictly observed.

The above GaN-LED design optimization is based on the popular assumption that electron leakage is the main reason for the efficiency reduction with rising current. However, other authors blame QW Auger recombination for this efficiency droop, which can be equally validated by LED simulations, even for the same device structure (Piprek 2015). Both carrier loss mechanisms can be reduced by lowering the QW carrier density. Rouet-Leduc et al. (2016) published such GaN-LED design optimization based on active machine learning, but without revealing much detail about LED parameters. Utilizing the APSYS software, the authors successively perform about 1000 simulations by varying the composition of their five InGaN quantum wells independently. The QW barrier composition is also changed and the QW thickness is adjusted to maintain a nearly constant emission wavelength. This strategy eventually leads to an IQE improvement from 0.55 to 0.78 at $75 \mathrm{~A} / \mathrm{cm}^{2}$ current density. Their machine learning algorithm finds the highest efficiency for widened QWs with about $11 \%$ indium sandwiched between InGaN barriers with $5 \%$ indium. This design optimization is quite intuitive as thicker QWs reduce the QW carrier density and shallower QWs shrink the built-in polarization field that separates electrons and holes inside the QW.

For evaluation, we apply this optimization strategy to the same LED reference structure simulated above (Tab. 1). Our IQE of 0.58 at $75 \mathrm{~A} / \mathrm{cm}^{2}$ (Fig. 4) is close to the value 0.55 reported by Rouet-Leduc et al. (2016) for their reference LED. The proposed addition of 5\% indium to the QW barriers results in the dashdot line in Fig. 4. The efficiency at $75 \mathrm{~A} / \mathrm{cm}^{2}$ drops due to enhanced electron leakage enabled by the lower $\mathrm{QW}$ barriers. Widening the QW from $3 \mathrm{~nm}$ to $5 \mathrm{~nm}$ causes a rising efficiency as the QW carrier density is reduced (dash-dot-dot line in Fig. 4). The final reduction of the QW indium content to $11 \%$ lowers the efficiency again due to stronger leakage from shallow QWs, despite a weaker polarization (dashed line in Fig. 4). The resulting band gap profile is shown as green line in Fig. 2. While the proposed design changes slightly enhance the peak efficiency at low current, IQE remains lower than with the reference LED at the target current density of operation $\left(75 \mathrm{~A} / \mathrm{cm}^{2}\right.$, see Fig. 4). Obviously, the success of this design optimization depends on more parameters than those considered by the authors. They skipped an essential step, namely the identification of the dominating loss mechanism in their simulation, which 
needs to be suppressed in the optimized design. Simulations should always investigate internal device physics first before developing design optimization strategies.

Rouet-Leduc (2017) applied similar machine learning methods to the fitting of simulated efficiency characteristics to a measured one. This approach accounts for well-known uncertainties of key material parameters: built-in polarization, recombination coefficients, QW carrier scattering time, and the exchange coefficient controlling the band-gap shrinkage with rising QW carrier density. Based on a large set of known simulation results for random material parameters, active machine learning is used to select material parameter sets for new simulations, thereby successively increasing the available data base. Reasonable agreement between measurement and simulation is achieved after about 300 iterations. While this is an important and valuable approach, there are two main shortcomings. First, the final fit parameters are not listed and discussed, i.e., this machine learning demonstration does not improve our understanding of device physics. Second, the time-consuming generation of hundreds of APSYS simulation results needs to start over for any design modification. A fitting procedure based on physical insight is probably faster and certainly more educating.

These examples demonstrate that simulation-based machine learning is extremely challenging due to the complexity of optoelectronic device physics. Reliable performance predictions are impossible without thorough validation of simulation models, including material parameters, and strict observance of design space limits. We here focus on problems related to LED physics and simulation, machine learning pitfalls are investigated elsewhere (Lipton \& Steinhardt 2018, Riley 2019, Kailkhura et al. 2019, Zunger 2019, Chugh et al. 2019, Heaven 2019). New scientific discoveries are unlikely as machine learning usually interpolates between known numerical results. This dilemma may be addressed in various ways, some of which are outlined in the following. The key element of such approaches is the involvement of experimental data.

Assumptions about the simulated device design are usually based on technological intentions and not on the actual structure fabricated, hindering a comparison between simulation and experiment. Typical examples are layer thickness, composition, and doping profile. Direct measurements of such structural data should be preferred in the simulation setup, whenever possible.

Material parameters are a main source of device simulation uncertainties. Big-data materials science could provide valuable input. For instance, the Materials Genome Initiative demonstrates a synergetic combination of theoretical models, computational methods, and experimental procedures (de Pablo et al. 2019, Lookman et al. 2019). Another example is the FAIR Data Initiative (Draxl \& Scheffler 2019). Such activities hopefully lead to more reliable models for key material properties relevant to optoelectronic device simulations. High-end models for sophisticated properties such as optical gain and absorption could be calculated separately and then imported into the full device simulation via ANN or other suitable means (Piprek et al. 2005). Parameter uncertainties should be considered in the simulation to enable a much-needed establishment of error ranges for calculated results (Piprek 2019). 
The initial disagreement between simulation results and performance measurements is often eliminated by adjusting material parameters in the model (Piprek et al. 2002). Each parameter usually requires a different measurement for calibration. The industrial fabrication of optoelectronic devices typically includes the automatic data acquisition from various measurements (Altermatt et al. 2018). Some values scatter significantly, which may be related to process variations (Wasmer \& Klöter 2019). Machine learning from measured data can be used to analyze such fluctuations, to extract material parameters (Majeed et al. 2020), or to select the experimental characteristics most appropriate for evaluating and calibrating simulations. Simulation-based digital twins of fabricated devices can provide a deeper understanding of how process variations influence device performance (Ibrahim et al. 2020, WagnerMohnsen \& Altermatt 2020). Generative adversarial networks (GANs) may be able to combine calculated and measured data in order to achieve more realistic predictions (Jiang et al. 2019, Yang et al. 2020, Kudyshev et al. 2021).

In summary, simulation-based machine learning is extremely challenging due to the complexity of optoelectronic device physics, which should be considered carefully. Simulations always simplify and potentially falsify reality. Without experimental validation of models and parameters, predictions of optoelectronic device performance remain unreliable. Employment of measured data in the computational analysis helps reducing the gap between theory and reality. Methods and results should be documented and scrutinized in detail to make publications credible, reproducible, and actionable.

\section{Declarations}

Funding: not applicable

Conflicts of interest/Competing interests: not applicable

Availability of data and material: not applicable

Code availability: not applicable

\section{References}

Altermatt, P., Yang, Y., Sheng, Y., Chen, D. et al.: A method for optimizing PERC cells in industrial production lines using final IV parameters, statistical procedures and numerical device modeling. AIP Conference Proceedings 1999, 110001 (2018). https://doi.org/10.1063/1.5049310

Chugh, S., Ghosh, S., Gulistan, A., Rahman, B.M.A.: Machine Learning Regression Approach to the Nanophotonic Waveguide Analyses. IEEE J. Lightw. Technol. 37, 6080-6089 (2019)

Cicic, S. \& Tomic, S.: Genetic algorithm designed high efficiency laser power converters based on the vertical epitaxial heterostructure architecture. Solar Energy Materials and Solar Cells 200, 109878 (2019) 
de Pablo, J.J., Jackson, N.E., Webb, M.A. et al.: New frontiers for the materials genome initiative. npj Comput Mater 5, 41 (2019)

Draxl, C. \& Scheffler, M.: The NOMAD laboratory: from data sharing to artificial intelligence. J. Phys.: Mater. 2, 036001 (2019)

Fan, J., Li, Y., Fryc, I., Qian, C., Fan, X., Zhang, G.: Machine-Learning Assisted Prediction of Spectral Power Distribution for Full-Spectrum White Light-Emitting Diode. IEEE Photon. J. 12, 8200218 (2020)

Genty, G., Salmela, L., Dudley, J.M. et al.: Machine learning and applications in ultrafast photonics. Nat. Photonics (2020). https://doi.org/10.1038/s41566-020-00716-4

Hakimian, F., Shayesteh, M.R., and Moslemi, M.R.: Optimization of a quantum-dot semiconductor optical amplifier (QD-SOA) design using the genetic algorithm. Opt Quant Electron 52, 48 (2020)

Heaven, D.: Why deep-learning Als are so easy to fool. Nature 574, 163-166 (2019)

Ibrahim, M. S., Fan, J., Yung, W. K. C., Prisacaru, A., van, W., Fan, X., Zhang, G.: Machine Learning and Digital Twin Driven Diagnostics and Prognostics of Light-Emitting Diodes. Laser \& Photonics Reviews 14, 2000254 (2020) . https://doi.org/10.1002/Ipor.202000254

Janai, M.A.B., Woon, K.L., Chan, C.S.: Design of efficient blue phosphorescent bottom emitting light emitting diodes by machine learning approach. Organ. Electron. 63, 257-266 (2018)

Jiang, J., Sell, D., Hoyer, S., Hickey, J., Yang, J., Fan, J.A.: Free-Form Diffractive Metagrating Design Based on Generative Adversarial Networks. ACS Nano 13, 8872-8878 (2019).

https://doi.org/10.1021/acsnano.9b02371

Kailkhura, B., Gallagher, B., Kim, S. et al.: Reliable and explainable machine-learning methods for accelerated material discovery. npj Comput Mater 5, 108 (2019)

Kim. D.Y., Lin, G.B., Hwang, S., Park, J. H., Meyaard, D., Schubert, E.F., Ryu, H.Y., Kim, J.K.: PolarizationEngineered High-Efficiency GaInN Light-Emitting Diodes Optimized by Genetic Algorithm. IEEE Photonics J. 7, 1300209 (2015)

Kudyshev, Z. A., Kildishev, A. V., Shalaev, V. M., Boltasseva, A.: Machine learning-assisted global optimization of photonic devices, Nanophotonics 10, 371-383 (2021). https://doi.org/10.1515/nanoph2020-0376

LeCunn, Y., Bengio, Y., Hinton, G.: Deep learning, Nature 521, 436-444 (2015)

Lipton, Z.C. \& Steinhardt, J.: Troubling Trends in Machine Learning Scholarship, ACM Queue 17, 1-33 (2019) https://doi.org/10.1145/3317287.3328534 
Liu, Z., Na, G., Tian, F., Yu, L., Li, J., Zhang, L.: Computational functionality-driven design of semiconductors for optoelectronic applications. InfoMat 2 (2020) . https://doi.org/10.1002/inf2.12099

Lookman, T., Balachandran, P.V., Xue, D. et al.: Active learning in materials science with emphasis on adaptive sampling using uncertainties for targeted design. npj Comput Mater 5, 21 (2019)

Lu, Q., Liu, Q., Sun, Q., Hsieh, C.Y., Zhang, S., Shi, L., Lee, C.K.: Deep Learning for Optoelectronic Properties of Organic Semiconductors, J. Phys. Chem. C 124, 7048-7060 (2020).

https://doi.org/10.1021/acs.jpcc.0c00329

Luo, S., Li, T., Wang, X., Faizan, M., Zhanf, L.: High-throughput computational materials screening and discovery of optoelectronic semiconductors. WIREs Comput. Mol. Sci. 11, e1489 (2020). https://doi.org/10.1002/wcms.1489

Ma, W., Liu, Z., Kudyshev, Z.A. et al.: Deep learning for the design of photonic structures. Nat. Photonics (2020). https://doi.org/10.1038/s41566-020-0685-y

Ma, Z. \& Li, Y.: Parameter extraction and inverse design of semiconductor lasers based on the deep learning and particle swarm optimization method. Opt. Express 28, 21971-21981 (2020)

Majeed, N., Saladina, M., Krompiec, M., Greedy, S., Deibel, C., MacKenzie, R. C. I.: Using Deep Machine Learning to Understand the Physical Performance Bottlenecks in Novel Thin-Film Solar Cells. Adv. Funct. Mater. 30, 1907259 (2020). https://doi.org/10.1002/adfm.201907259

Molesky, S., Lin, Z., Piggott, A. Y., Jin, W., Vuckovic, J., Rodriguez, W.: Inverse design in nanophotonics. Nature Phot. 12, 659-670 (2018) 659

Müller, M., Altermatt, P.P., Wagner , H., Fischer, G.: Sensitivity Analysis of Industrial Multicrystalline PERC Silicon Solar Cells by Means of 3-D Device Simulation and Metamodeling. IEEE J. Photovolt. 4, 107-113 (2014)

Piprek, J., White, J.K., SpringThorpe, A.J.: What limits the maximum output power of long-wavelength AlGalnAs/InP laser diodes? IEEE J. Quantum Electron. 38, 1253-1259 (2002)

Piprek, J., Li, S., Mensz, P., Hader, J: Monolitic Wavelength Converter: Many-Body Effects and Saturation Analysis, Ch. 14 in Optoelectronic Devices: Advanced Simulation and Analysis, Piprek, J. (ed.), pp. 405546, Springer, New York (2005)

Piprek, J. \& Li, S.: GaN-based Light-Emitting Diodes, Ch. 10 in Optoelectronic Devices: Advanced Simulation and Analysis, Piprek, J. (ed.), pp. 293-312, Springer, New York (2005)

Piprek, J.: Efficiency droop in nitride-based light-emitting diodes. Phys. Status Solidi A 207, 2217- 2225 (2010) 
Piprek, J. \& Li, S.: Electron leakage effects on GaN-based light-emitting diodes. Opt. Quant. Electron. 42, 89-95 (2010)

Piprek, J. \& Li, Z.M.: Sensitivity analysis of electron leakage in III-nitride light-emitting diodes. Appl. Phys. Lett. 102, 131103 (2013)

Piprek, J., Römer, F., Witzigmann, B: On the uncertainty of the Auger recombination coefficient extracted from InGaN/GaN light-emitting diode efficiency droop measurements. Appl. Phys. Lett. 106, 101101 (2015)

Piprek, J.: How to decide between competing efficiency droop models for GaN-based light-emitting diodes. Appl. Phys. Lett. 107, 031101 (2015)

Piprek, J. (ed.), Handbook of optoelectronic device modeling and simulation, CRC Press, Boca Raton (2017)

Piprek, J. On the reliability of pulse power saturation models for broad-area GaAs-based lasers. Opt Quant Electron 51, 60 (2019)

Piprek, J.: Efficiency Models for GaN-based Light Emitting Diodes: Status and Challenges, MDPI Materials $13,5174(2020)$

Razzaq, A., Mayer, A., Depauw, V., Gordon, I., Hajjiah, A. and Poortmans, J., Application of a Genetic Algorithm in Four-Terminal Perovskite/Crystalline-Silicon Tandem Devices. IEEE J. Photovolt. 10, 1689 $1700(2020)$

Riley, P.: Three pitfalls to avoid in machine learning. Nature 572, $27-29$ (2019)

Rouet-Leduc, B., Barros, K., Lookman, T. , Humphreys, C. J.: Optimization of GaN LEDs and the reduction of efficiency droop using active machine learning. Sci Rep 6, 24862 (2016)

Rouet-Leduc, B.: Machine learning for materials science. Ph.D. Thesis, Univ. of Cambridge (2017)

Schmidt, J., Marques, M.R.G., Botti, S., Marques, M.A.L.: Recent advances and applications of machine learning in solid-state materials science. npj Comp. Mat. 5, 83 (2019)

Usman, M., Anwar, A.R., Munsif, M.: A Survey of Simulations on Device Engineering of GaN-Based LightEmitting Diodes. ECS J. Solid State Sci. Technol. 9, 066002 (2020)

Verzellesi, G., Saguatti, D., Meneghini, M., Bertazzi, F., Goano, M., Meneghesso, G., Zanoni, E.: Efficiency droop in InGaN/GaN blue light-emitting diodes: Physical mechanisms and remedies. Appl. Phys. Rev. 114, 071101 (2013)

Wagner-Mohnsen, H. \& Altermatt, P.P.: A Combined Numerical Modeling and Machine Learning Approach for Optimization of Mass-Produced Industrial Solar Cells. IEEE J. Photovolt.10, 1441-1447 (2020) 
Wasisto, H.S., Prades, J.D., Gulink, J., Waag, A.: Beyond solid-state lighting: Miniaturization, hybrid integration, and applications of GaN nano- and micro-LEDs. Appl. Phys. Rev. 6, 041315 (2019)

Wasmer, S., Greulich, J., Höffler, H., Wöhrle, N., Demant, M., Fertig, F., Rein, S.: Impact of Material and Process Variations on the Distribution of Multicrystalline Silicon PERC Cell Efficiencies. IEEE J. Photovolt. 7, 118-128 (2017)

Wasmer, S. \& Klöter, B.: Interpretable Machine Learning for Production Optimization. Proc. $36^{\text {th }}$ EUPVSEC, 272 (2019). https://doi.org/10.4229/EUPVSEC20192019-2D0.5.6

Yang, L., Zhang, D., Karniadakis, G. E., Physics-Informed Generative Adversarial Networks for Stochastic Differential Equations. SIAM Journal on Scientific Computing, 42, A292-A317 (2020)

Zhu, D., Schubert, M. F., Cho, J., Schubert, E.F., Crawford, M.H., Koleske, D.D., Shim, H., Sone, C.: Genetic Algorithm for Innovative Device Designs in High-Efficiency III-V Nitride Light-Emitting Diodes. Appl. Phys. Expr. 5, 012102 (2012)

Zunger, A.: Beware of plausible predictions of fantasy materials. Nature $566,447-449$ (2019)

\section{Figures}




\section{theory}

computer simulation (deductive)

\section{virtual reality}

\section{statistics}

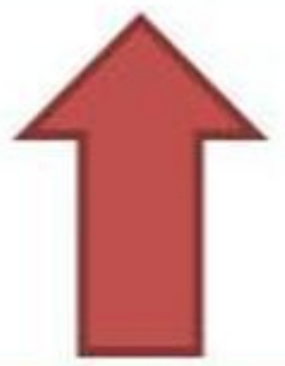

machine learning

(inductive)

\section{reality}

Figure 1

Deductive vs. inductive method. 


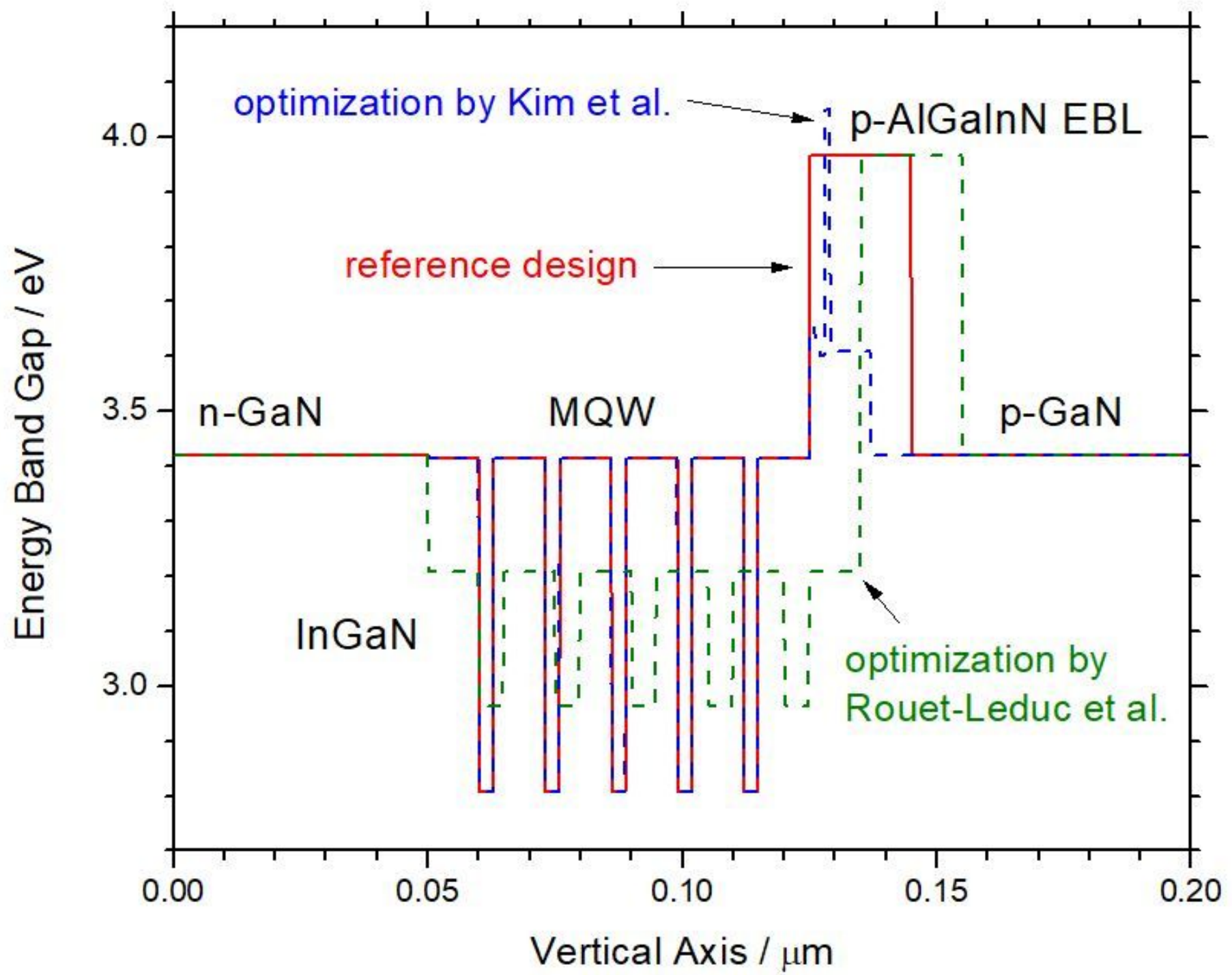

Figure 2

Comparison of LED band gap profiles (MQW - multiple quantum well, EBL - electron blocking layer). 


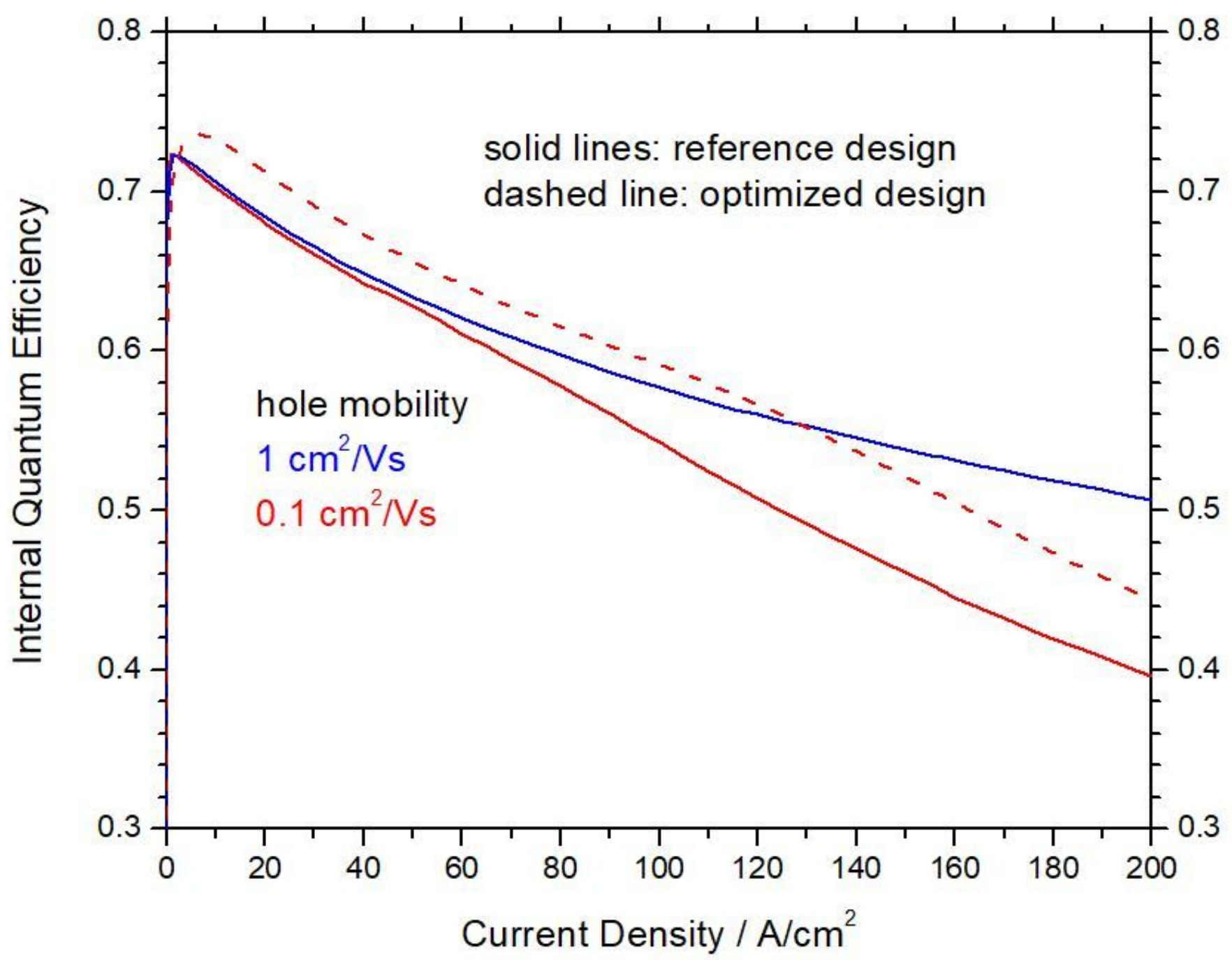

Figure 3

LED efficiency simulation for designs by Kim et al. (2015) 


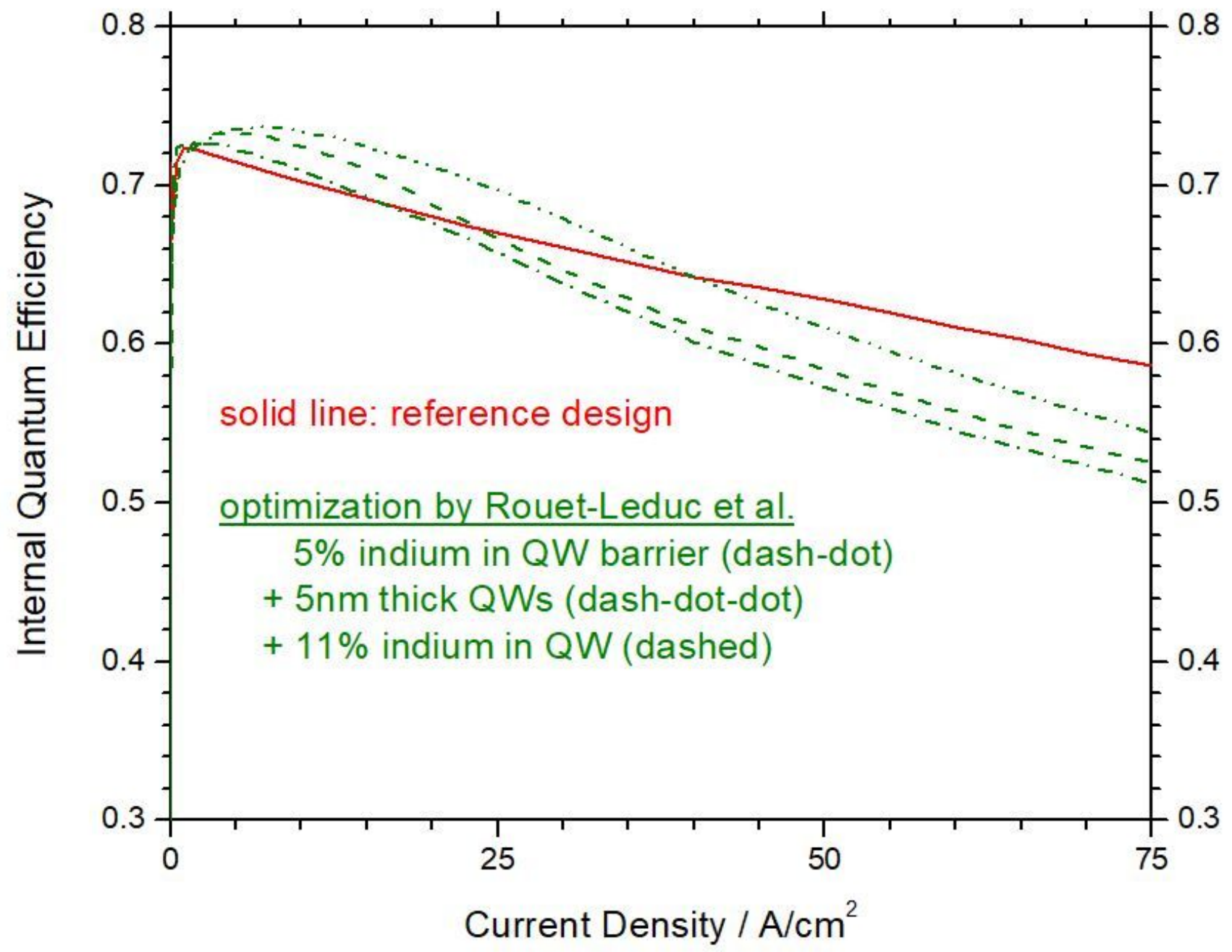

Figure 4

LED efficiency simulation of the design changes proposed by Rouet-Leduc et al. (2016) 